\title{
Radiation Resistance and Gain of Homogeneous Ring Quasi-Array
}

\author{
Knudsen, H. L.
}

Published in:

Proceedings of the IEEE

Link to article, DOI:

10.1109/JRPROC.1954.274705

Publication date:

1954

Document Version

Publisher's PDF, also known as Version of record

Link back to DTU Orbit

Citation (APA):

Knudsen, H. L. (1954). Radiation Resistance and Gain of Homogeneous Ring Quasi-Array. Proceedings of the IEEE, 42(4), 686-695. https://doi.org/10.1109/JRPROC.1954.274705

\section{General rights}

Copyright and moral rights for the publications made accessible in the public portal are retained by the authors and/or other copyright owners and it is a condition of accessing publications that users recognise and abide by the legal requirements associated with these rights.

- Users may download and print one copy of any publication from the public portal for the purpose of private study or research.

- You may not further distribute the material or use it for any profit-making activity or commercial gain

- You may freely distribute the URL identifying the publication in the public portal

If you believe that this document breaches copyright please contact us providing details, and we will remove access to the work immediately and investigate your claim. 
The new voltage vector after interconnection is found $\mathrm{E}_{2}=\left(\mathrm{C}_{2}\right)_{t} \mathrm{E}_{1}$ as

$$
\begin{array}{rlll|lllllllll}
\mathbf{j} & \mathbf{m} \\
\mathrm{E}_{2}=\frac{E_{A \boldsymbol{s}}}{E_{B e}} & E_{C e} & E_{D e} & 0 & 0 & 0 & 0 & 0 & 0 & 0 & 0
\end{array}
$$

That is, all mesh voltages become zero, since all ground points are short-circuited.

\section{Elimination of Meshes (Frame 3)}

The unknown 8 mesh currents $\mathbf{i}$ are eliminated by ${ }^{13}$

$$
Z_{22}{ }^{\prime}=Z_{1}-Z_{2} Z_{4}{ }^{-1} Z_{3},
$$

giving the solution of the resultant system of Fig. 7 as $\mathbf{E}_{3}=\mathbf{Z}_{33} \mathbf{I}^{3}$. There are only four scalar equations, representing the voltages $\mathbf{E}$ across the four junctions at which I is impressed.

\section{Solving for the Mesh Currents}

Since the component matrices $\mathbf{Z}_{1}, \boldsymbol{Z}_{2}$ and $\boldsymbol{Z}_{3}$ of (9) are strongly diagonal with many zero off-diagonal elements, it is preferable not to perform the elimination, but to leave (8) in its given form. That is the solution of $I=Y E$ is represented by the form

$$
\mathbf{E}=\left(\mathbf{Z}_{1}-\mathbf{Z}_{2} \mathrm{Y}^{4} \mathbf{Z}_{3}\right) \mathrm{I} \text {. }
$$

${ }^{13}$ Ibid., p. 61.
For any given numerical I, the substitutions indicated in (11) are to be performed in succession.

The "orthogonal" inverse table may be represented as

$$
Z_{22}^{\prime}=\left|\begin{array}{ll}
Z_{1} & Z_{2} \\
Z_{3} & Z_{4}^{-1}
\end{array}\right|
$$

differing from (9) by the presence of the constraint (mesh current) inverse $\mathrm{Z}_{4}^{-1}=\mathrm{Y}^{4}$. The "orthogonal" inverse table is strongly diagonal and contains only a small fraction of the nonzero elements that exist in the conventional inverse matrix.

Thus, another important consequence of the process of tearing is that it requires far less computation to solve simultaneously for both forces and constraints, than to solve for the forces alone.

\section{Calculation of Junction Potentials}

In the example shown only some of the junctions have a current $I$ impressed upon them. It was also assumed that the potentials $E$ of those junctions that have no $I$, are of no interest. If the potentials of all junctions have to be known, then the number of assumed junction pairs in Fig. 4(a) is 8 instead of 5 and $\left(\mathbf{C}_{2}{ }^{1}\right)_{t}$ assumes more columns. The steps are analogous to those given elsewhere. ${ }^{14}$

${ }^{14}$ Ibid., p. 128.

\section{Radiation Resistance and Gain of Homogeneous Ring Quasi-Array*}

H. L. KNUDSEN $\dagger$

\begin{abstract}
Summary - In a previous paper homogeneous ring quasi-arrays of tangential or radial dipoles were introduced, i.e. systems of dipoles arranged equidistantly along a circle, the dipoles being oriented in tangential or radial directions and carrying currents with the same amplitude, but with a phase that increases uniformly along the circle. Such quasi-arrays are azimuthally omnidirectional, and the radiated field will be mainly horizontally polarized and concentrated around the plane of the circle. In this paper expressions are obtained for the radiation resistance and the gain of homogeneous ring quasi-arrays of an infinite number of tangential or radial dipoles, and from the results arrived at conclusions are drawn as to the applicability of such antenna systems.
\end{abstract}

* Decimal classification: R-129. Original manuscript received by the Institute, August 28,1953 . The work described here was carried out at The Microwave Laboratory of The Royal Technical University of Denmark, Copenhagen, Denmark, and was made possible through the support granted by the Research Council of the Technical Sciences in Denmark. It forms part of a Doctor's thesis ("Bidrag til teorien for antennesystemer med hel eller delvis rotationssymmetri," 228 pp., Copenhagen, Denmark, 1953). Some sections of this thesis have already been published in American journals.

† The Microwave Laboratory, The Royal Technical University of Denmark, Copenhagen, Denmark.

\section{INTRODUCTION}

TN A PREVIOUS PAPER ${ }^{1}$ it was shown that an azimuthally omnidirectional antenna system with a field that is concentrated around the horizontal plane and with predominantly horizontal polarization may be obtained by placing equidistantly along a circle in the horizontal plane a number of tangential or radial dipoles and by feeding these dipoles with currents having the same numerical value but a phase that increases uniformly along the circle. An antenna system of this type will be called a homogeneous ring quasi-array of tangential or radial dipoles. In order that this ring quasi-array may be completely omnidirectional in the horizontal plane the number of dipoles must be infinitely large; this case is illustrated in Figs. 1(b) and

${ }^{1} \mathrm{H}$. L. Knudsen, "The field radiated by a ring quasi-array of an infinite number of tangential or radial dipoles," PROC. I.R.E., vol. 41, pp. 781-789; June, 1953. 
7(b). The current phase increases a whole number, $H$, times $2 \pi$ during one revolution in the positive direction. When the increment of the current phase along the circle augments, the radiation pattern concentrates to an increasing degree into a narrow, disk-shaped bundle around the horizontal plane. At the same time the vertical component of the electric field strength decreases in relation to the horizontal component. These properties of homogeneous ring quasi-arrays of tangential or radial dipoles explain their practical applicability.

Expressions for the field components and a thorough discussion of the radiation pattern of homogeneous ring quasi-arrays are given in the above mentioned paper. ${ }^{1}$ However, with a view to the complete understanding and description of these antenna systems it is desirable to have expressions for the radiation resistance and the gain of these antenna systems. The present paper is devoted to the derivation and discussion of such expressions. In order to simplify the calculations the number of dipoles in each ring quasi-array is assumed to be infinitely large; this simplifying assumption should not cause any appreciable deviation from the actual conditions.

The special case of a ring quasi-array of tangential dipoles with constant current phase, i.e. $H=0$, has been dealt with in detail by Foster, ${ }^{2}$ who applies the Poynting vector method, and by Moullin, ${ }^{3}$ who applies the emf method. The results reached by these authors are contained as special cases in the present investigation.

The radiation resistance and the gain in the case of an arbitrary value of $H$ will be derived here on the basis of the Poynting vector method. The calculations follow a line similar to that of Foster ${ }^{2}$ in his calculations for the special case $H=0$. However, Foster's method has to be modified and extended in order that it may be applicable here. To some extent Page's ${ }^{4}$ calculation of the radiation resistance and gain of a homogeneous ring array of axial dipoles has also been guiding in the following calculations.

\section{Tangential Dipoles}

\section{Calculation of Radiation Resistance and Gain}

In this section we shall calculate the radiation resistance and the gain of a homogeneous ring quasi-array of an infinite number of dipoles placed along a circle with radius $a$ so that the dipoles are tangent to this circle, as shown in Fig. 1(b). We first formulate the problem of the corresponding ring quasi-array with a finite number, $s$, of elements, each one having the length $L$, see Fig. 1(a). As shown in this figure, we introduce a spherical co-ordinate system $(r, \theta, \phi)$. In this system the $j^{\prime}$ th dipole has the co-ordinates

2 D. Foster, "Loop antennas with uniform current," Proc. I.R.E., vol. 32, pp. 603-607; October, 1944.

${ }^{3}$ E. B. Moullin, "Radiation from large circular loops," Jour. IEE part III, vol. 93, pp. 345-351; 1946.

${ }^{4} \mathrm{H}$. Page, "Radiation resistance of ring aerials," Wireless Eng., vol. 25, pp. 102-109; April, 1948.
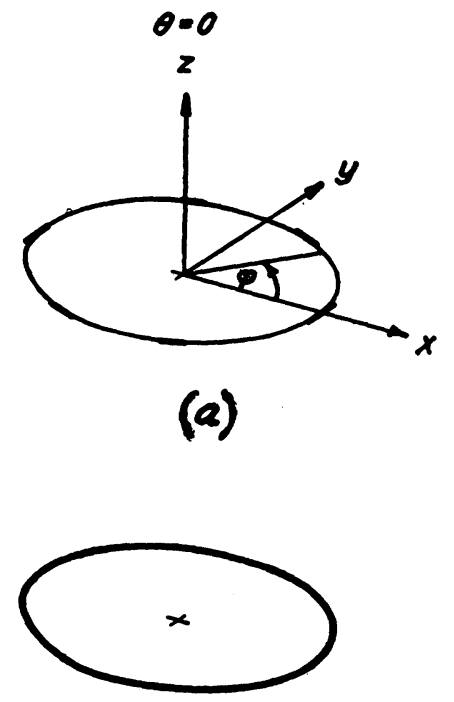

(b)

Fig. 1-Ring quasi-array of: (a) a finite number of tangential dipoles, and (b) infinitely many tangential dipoles.

$$
(r, \theta, \phi)_{j}^{\prime} \text { th dipole }=\left(a, \frac{\pi}{2}, u_{j}\right)
$$

where

$$
u_{j}=\frac{2 \pi j}{s}, \quad j=1,2, \cdots, s .
$$

The current $I_{j}$ in the $j^{\prime}$ th dipole is given by

$$
I_{j}=I e^{i H u_{i}},
$$

where $I$ is complex constant and $H$ integral number.

We now let the number of elements, $s$, increase beyond all limits and the length of each dipole, $L$, decrease in such a way that the product $s L$ remains finite. As shown in a previous paper, ${ }^{1}$ the components of the electric field strength in the far zone field of the quasiarray are then expressed by

$$
\bar{E}=T \bar{F},
$$

where

$$
T=\frac{i k s L \zeta I}{4 \pi r} e^{i k r}
$$

and

$$
\begin{aligned}
F_{\theta} & =\frac{H}{z} J_{H}(z) \cos \theta e^{i H(\phi-\pi / 2)}, \\
F_{\phi} & =i J_{H}^{\prime}(z) e^{i H(\phi-\pi / 2)}, \\
z & =k a \sin \theta .
\end{aligned}
$$

In this expression $k=\omega \sqrt{\mu \epsilon}$ denotes the intrinsic propagation constant and $\zeta=\sqrt{\mu / \epsilon}$ the intrinsic impedance of space. As an example we have plotted in Fig. 2 the radiation pattern of a quasi-array with $a / \lambda=0.6$ and $H=4$. 


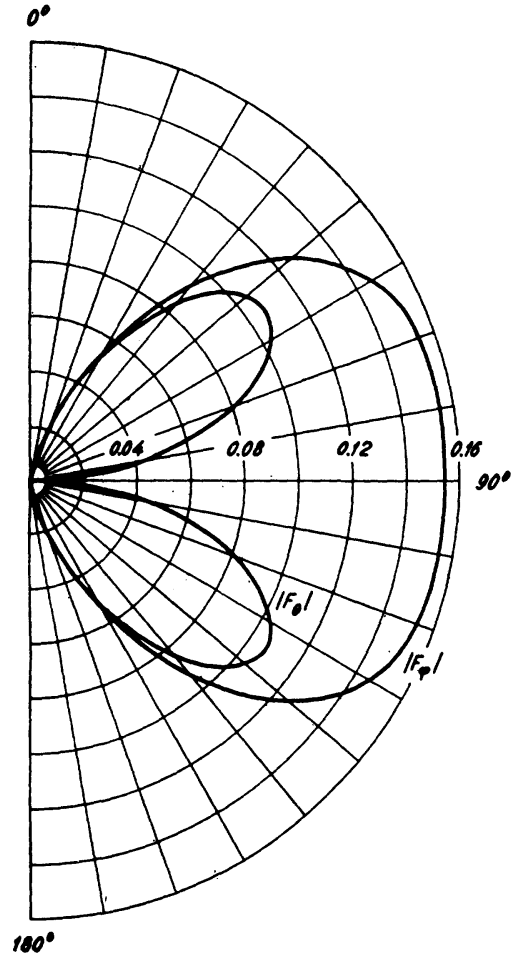

Fig. 2-Field $\theta$ - and $\phi$-components, radiated by a homogeneous ring quasi-array of tangential dipoles, with $\alpha / \lambda=0.6$ and $H=4$.

We now find the total radiated effect $P_{H}$ by integrating radial component of Poynting vector $\bar{S}=\frac{1}{2} \bar{E} \times \tilde{H}$ over a large spherical surface with radius $r$ and with its center at the antenna system. Hereby we obtain

$$
\begin{aligned}
P_{H} & =2 \pi \int_{0}^{\pi} S_{r} r^{2} \sin \theta d \theta \\
& =2 \pi \int_{0}^{\pi} \frac{r^{2}}{2 \zeta} \bar{E} \cdot \tilde{\bar{E}} \sin \theta d \theta=\frac{(k s L)^{2} \zeta I \tilde{I}}{8 \pi} M_{H}
\end{aligned}
$$

where

$$
M_{H}(k a)=\frac{1}{2} \int_{0}^{\pi} m_{H}(k a, \theta) \sin \theta d \theta
$$

with

$$
\begin{aligned}
m_{H}(k a, \theta)= & F_{\theta} \widetilde{F}_{\theta}+F_{\phi} \widetilde{F}_{\phi} \\
& =\left(\frac{H}{z} J_{H}(z) \cos \theta\right)^{2}+\left(J_{H}^{\prime}(z)\right)^{2} .
\end{aligned}
$$

In these and the following formulas $\tilde{a}$ denotes the complex conjugate of $a$.

Defining the radiation resistance $R_{H}$ in relation to the current $I$ in one of the dipoles by

$$
P_{H}=\frac{1}{2} R_{H} I \tilde{I}
$$

we finally find

$$
R_{H}=Z M_{H}(k a)
$$

where

$$
Z=\frac{(k s L)^{2}}{4 \pi} \zeta
$$

The directive properties of an antenna is expressed by its gain. The gain $g_{H}(\theta)$ of an azimuthally omnidirectional antenna in the direction $(\theta, \phi)$ is defined by

$$
g_{H}(\theta)=\frac{S(\theta)}{S_{\text {mean }}} .
$$

As

$$
S_{\text {mean }}=\frac{P_{H}}{4 \pi r^{2}}
$$

we find for the quasi-array considered here

$$
g_{H}(\theta)=\frac{m_{H}(k a, \theta)}{M_{H}(k a)},
$$

where $m_{H}(k a, \theta)$ and $M_{H}(k a)$ are expressed by (10) and (9), respectively.

The problem of calculating the radiation resistance as well as that of calculating the gain have thus been reduced to the question of obtaining an expression for the definite integral $M_{H}(k a)$. We have to investigate separately the cases $H=0$ and $|H| \geqq 1$.

$H=0$ : The definite integral $M_{0}(k a)$ has been evaluated by Foster. ${ }^{2} \mathrm{He}$ arrives at the result

$$
M_{0}(k a)=\frac{1}{2 k a} \bar{J}_{2}(2 k a),
$$

where we have introduced the notation

$$
\bar{J}_{n}(x)=\int_{0}^{x} J_{n}(t) d t,
$$

a notation which is used by several authors, though not by Foster.

The function $\bar{J}_{n}(x)$ has been tabulated for $n=0$, amongst others by Lowan and Abramowitz, ${ }^{5}$ who have published a table of $\bar{J}_{0}(x), 10 d, x=0(0.01) 10$. However, no table of $\bar{J}_{n}(x)$ seems to have been published for $n \geqq 1$, although these functions have found application also in the solution of other technical problems than the one considered here, e.g. in the calculation of transient phenomena in electric wave filters. ${ }^{6}$ The author has prepared a table of $\bar{J}_{n}(x), 5 d, n=1(1) 8$, $x=0(0.02) 10$, which is included in a recently published monograph. ${ }^{7}$ In Appendix I to the present paper the calculation of $\bar{J}_{n}(x)$ is briefly outlined, and curves of these functions are given.

By using the above mentioned table or the curves in Appendix I we may now calculate the definite integral $M_{0}(k a)$ as expressed by (17). The function $M_{0}(x)$ is plotted in Fig. 3 for $0 \leqq x \leqq 5$.

5 A. N. Lowan and M. Abramowitz, "Table of the integrals $\int_{0}^{x} J_{0}(t) d t$ and $\int_{0}^{x} Y_{0}(t) d t$," Jour. Math. Phys., vol. 22, pp. 2-12; 1943.

6 J. Rybner, "Om Heavisides Operatorregning," ("On Heaviside's operational calculus"), Ingeniфren (Copenhagen), part E, vol. 49, pp. 93-103; 1940.

7 H. L. Knudsen, "Bidrag til teorien for antennesystemer med hel eller delvis rotationssymmetri," (contribution to the theory of antenna systems with complete or in part rotational symmetry). I kommission hos Teknisk Forlag, Copenhagen, Denmark; 1953. 


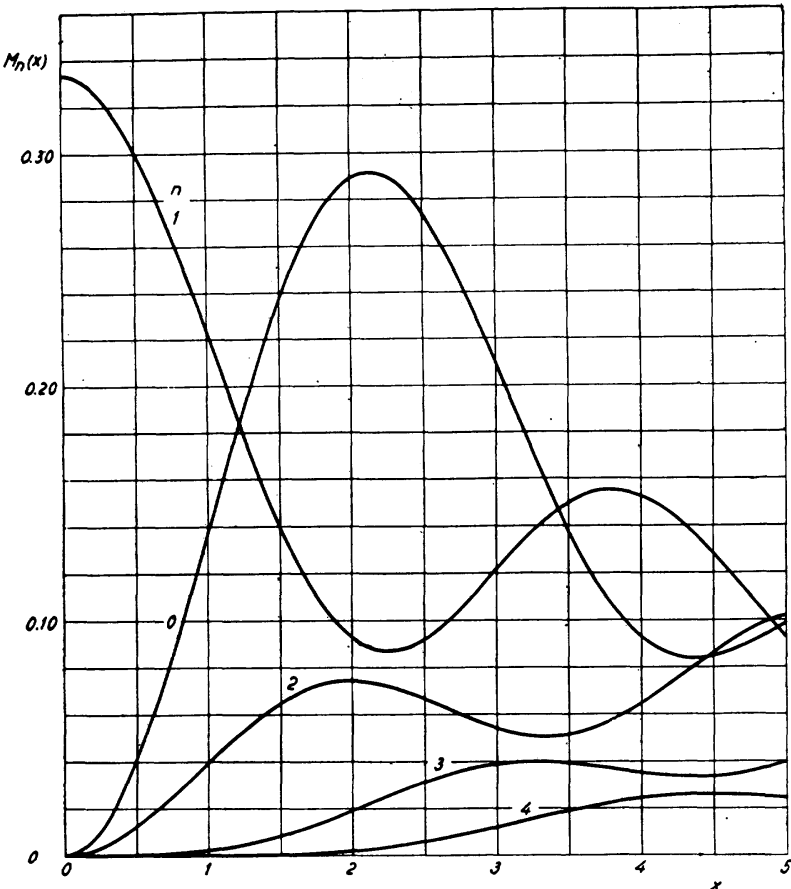

Fig. 3-The function $M_{n}(x)$ defined in (19) for a ring quasi-array of tangential dipoles.

$|H| \geqq 1$ : Having treated the case of $H=0$ we now assume that $|H| \geqq 1$. The radiation resistance and the gain being the same for $H$ and for $-H$, we need in this section only consider the positive values of $H$, i.e. we may assume that $H \geqq 1$. By inserting the expression (10) for $m_{H}$ in the expression (9) for $M_{H}$, applying Bessel's recursion formulas, and transforming further, we obtain

$$
\begin{aligned}
M_{H}(k a)= & \frac{1}{8} \int_{0}^{\pi}\left[\left(J_{H-1}(z)+J_{H+1}(z)\right)^{2} \cos ^{2} \theta\right. \\
& \left.+\left(J_{H-1}(z)-J_{H+1}(z)\right)^{2}\right] \sin \theta d \theta \\
= & \frac{1}{8} \int_{0}^{\pi}\left[\left(J^{2}{ }_{H-1}(z)+J^{2}{ }_{H+1}(z)\right)\left(1+\cos ^{2} \theta\right)\right. \\
& \left.-2 J_{H-1}(z) J_{H+1}(z) \sin ^{2} \theta\right] \sin \theta d \theta \\
= & \frac{1}{8}\left[A_{H-1}(k a)+A_{H+1}(k a)-2 B_{H}(k a)\right],
\end{aligned}
$$

where

$$
\begin{aligned}
& A_{n}(x)=\int_{0}^{\pi} J_{n}^{2}(x \sin \theta)\left(2-\sin ^{2} \theta\right) \sin \theta d \theta \\
& B_{n}(x)=\int_{0}^{\pi} J_{n-1}(x \sin \theta) J_{n+1}(x \sin \theta) \sin ^{3} \theta d \theta .
\end{aligned}
$$

The problem has now been reduced to the question of calculating the functions $A_{n}(x)$ and $B_{n}(x)$. In Appendix II it is shown that $A_{n}(x)$ and $B_{n}(x)$ may be expressed as follows

$$
\begin{aligned}
A_{n}(x)= & {\left[\frac{3}{2 x}-\frac{4 n^{2}-1}{8 x^{3}}\right] \bar{J}_{2 n}(2 x) } \\
& +\frac{2 n-1}{4 x^{2}} J_{2 n}(2 x)-\frac{1}{2 x} J_{2 n+1}(2 x),
\end{aligned}
$$

$$
\begin{aligned}
B_{n}(x)= & {\left[-\frac{1}{2 x}+\frac{3\left(4 n^{2}-1\right)}{8 x^{3}}\right] \bar{J}_{2 n}(2 x) } \\
& -\frac{3(2 n-1)}{4 x^{2}} J_{2 n}(2 x)+\frac{3}{2 x} J_{2 n+1}(2 x) .
\end{aligned}
$$

The functions $A_{n}(x)$ and $B_{n}(x)$, and consequently also the definite integral $M_{H}$, are now expressed through known functions, including the function $\bar{J}_{n}(x)$, which was defined in (18) and plotted in Appendix I.

The function $A_{n}(x)$ is plotted in Figs. 14 and 15. On the basis of these curves the function $M_{n}(x)$ has been calculated and plotted in Fig. 3 for $0 \leqq x \leqq 5$ and for $n=1,2,3$, and 4 .

\section{Discussion of the result}

We have now obtained expressions for the definite integral $M_{H}(k a)$ for any value of $H$. By inserting these expressions for $M_{H}(k a)$ in (12) we may now calculate the radiation resistance $R_{H}$. In Fig. $4(2 \pi a / s L)^{2} R_{H}$ is plotted as a function of $a / \lambda$ for $|H|=0,1,2,3$, and 4 . The radiation resistance corresponding to $H=0$ has been plotted in Foster's ${ }^{2}$ as well as in Moullin's ${ }^{3}$ papers

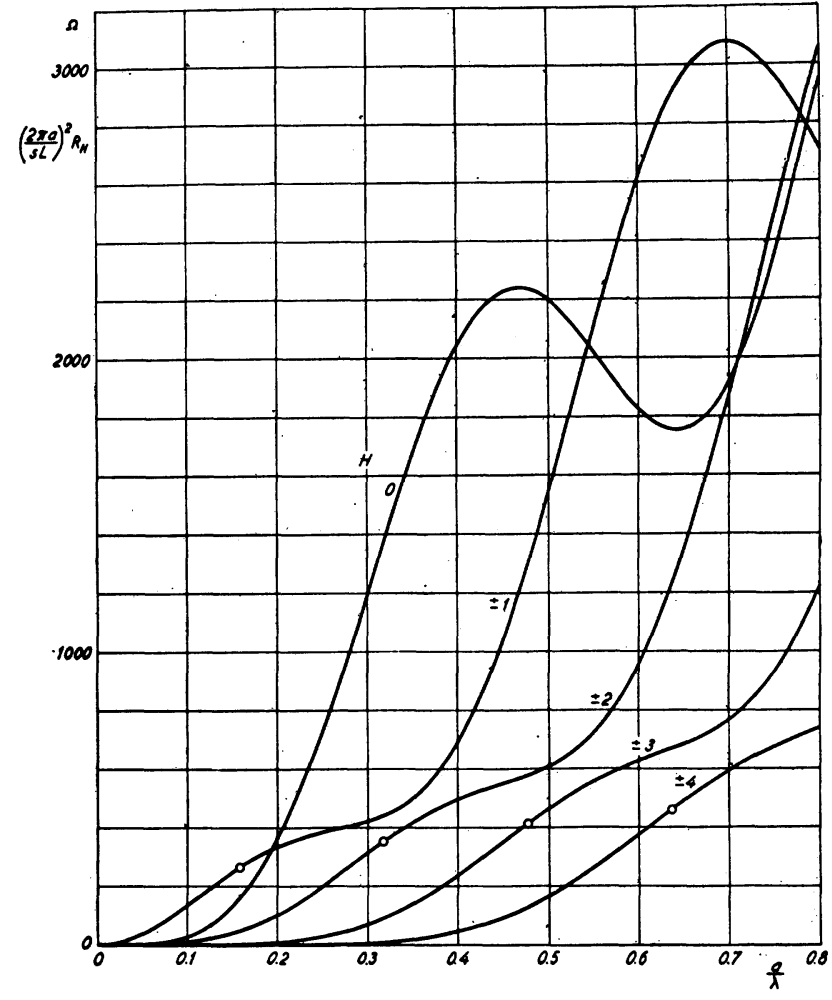

Fig. 4-The radiation resistance $R_{H}$ multiplied by $(2 \pi a / s L)^{2}$ of a homogeneous ring quasi-array of tangential dipoles.

previously referred to. It seems that an error has slipped into Moullin's calculation of the radiation resistance for $H=0$; as far as I can figure out, the radiation resistance is twice the magnitude given by the curve in Fig. 3 of Moullin's paper. The curves in Fig. 4 show that the radiation resistance for an arbitrary value of $H$ increases, though not monotonously, with increasing radius of the circle. For a constant radius the radiation 
resistance is seen to decrease with increasing $|H|$; when $|H|$ becomes sufficiently large, super-gain occurs, and the quasi-array becomes practically inapplicable, as was discussed in a former paper. ${ }^{8}$

By inserting the obtained expressions for $m_{H}(k a, \theta)$ and $M_{H}(k a)$ in (16) the gain $g(\theta)$ of the quasi-array may be found. The gain in horizontal directions, $\theta=\pi / 2$, is plotted in Fig. 5 as a function of $a / \lambda$ for $|H|=0,1,2,3$, and 4 . The curve corresponding to $H=0$ is plotted also in the above mentioned papers by Foster ${ }^{2}$ and Moullin ${ }^{3}$ gives for $H=0$ a curve showing the gain in the direction in which the gain assumes its largest value. For small values of the radius Foster's curve is identical with the curve in Fig. 5 that corresponds to $H=0$. Fig. 5 shows that the gain in horizontal directions for any value of $H$ first decreases monotonously towards zero and then oscillates. For $|H| \geqq 1$ and for small values of the radius the gain is seen to increase with increasing values of $|H|$. However, it is interesting to observe that for $|H|=1$ as well as for $|H|=2$, the gain-for small values of the radius-is smaller than the gain for $H=0$. Not until for $|H|=3$ the gain is larger than for $H=0$. As a consequence of the increase of the gain with increasing $|H|$ the homogeneous ring quasi-array of tangential dipoles should be useful as an azimuthally omnidirectional antenna system with a mainly horizontally polarized field concentrated around the horizontal plane.

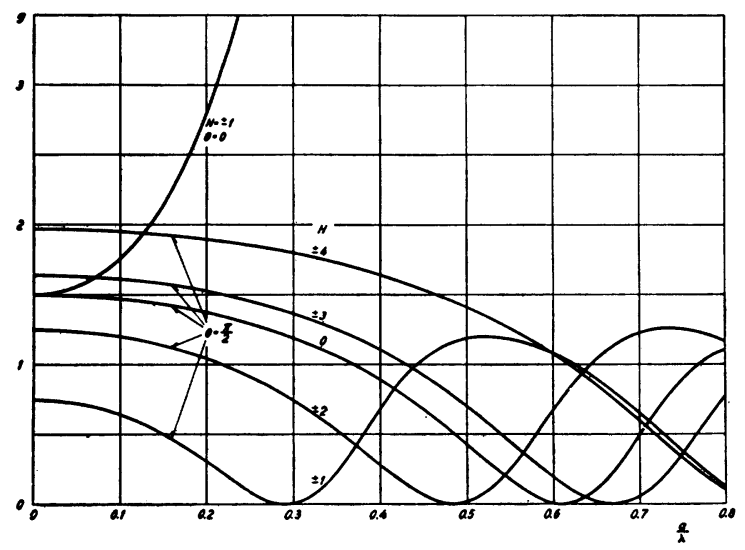

Fig. 5-The gain $g_{H}$ in horizontal directions of a homogeneous ring quasi-array of tangential dipoles. The figure also shows the gain in the vertical direction of a quasi-array of this type with $|H|=1$.

In Fig. 5 has been plotted for $|H|=1$, also the gain in the axial directions $\theta=0$ and $\pi$ as a function of $a / \lambda$. This is shown better in Fig. 6 . For other values of $H$ the gain in these directions is zero. Whereas the gain in horizontal directions for the values of $H$ and $a / \lambda$ occurring in Fig. 5 was smaller than 2 , the gain in the direction of the axis in the case of $|H|=1$ assumes much larger values. Its first maximum occurs for $a / \lambda=0.360$; the gain is then 5.75. As a consequence of its large gain in the axial directions a ring quasi-array of tangential dipoles

${ }^{8}$ H. L. Knudsen, "Superforstærkning hos antenner" ("Super-gain of antennas"). Elektroteknisk Tidsskrift (Oslo, Norway), vol. 64, pp. $213-221 ; 1951$. with $|H|=1$ should be useful as a bilaterally radiating beam antenna system with circular polarization in the directions of the principal lobes.

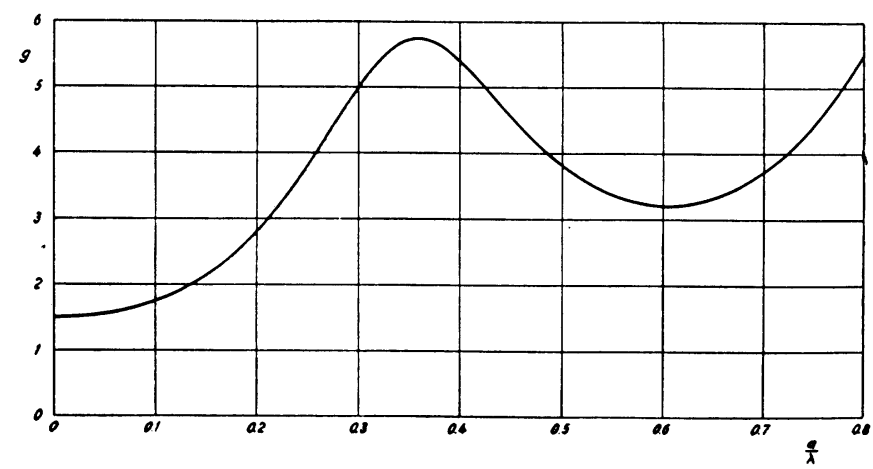

Fig. 6-The gain $g_{1}$ in the vertical direction of a homogeneous ring quasi-array of tangential dipoles with $|H|=1$.

\section{Radial Dipoles}

\section{Calculation of Radiation Resistance and Gain}

In this section we shall carry out a similar calculation of the radiation resistance and the gain of a homogeneous ring quasi-array of an infinite number of dipoles placed along a circle with radius $a$ and oriented in radial directions, as shown in Fig. 7(b). We first formulate the problem of the corresponding ring quasi-array with a finite number, $s$, of elements, each one having the length $L$, see Fig. 7 (a). In a spherical co-ordinate system $(r, \theta, \phi)$, as shown in the figure, the position of the $j^{\prime}$ th dipole may be expressed by (1) and (2), and the current in the $j^{\prime}$ th dipole by (3).

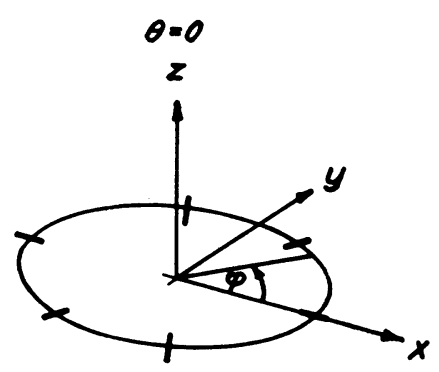

(a)

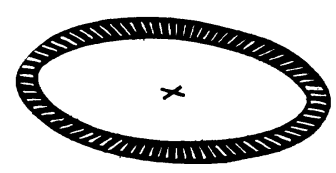

(b)

Fig. 7-Ring quasi-array of: (a) a finite number of radial dipoles, and (b) infinitely many radial dipoles.

We let now the number of elements, $s$, increase towards infinity and the length of each dipole, $L$, decrease towards zero in such a way that $s L$ remains finite. As was shown in a previous paper ${ }^{1}$ the electric field strength in the far zone may then be expressed by (4) and (5), 
where

$$
\begin{aligned}
& F_{\theta}=i J_{H}{ }^{\prime}(z) \cos \theta e^{i H(\phi-\pi / 2)}, \\
& F_{\phi}=-\frac{H}{z} J_{H}(z) e^{i H(\phi-\pi / 2)},
\end{aligned}
$$

where $z$ is given by (7). As an example the radiation pattern of a quasi-array with $a / \lambda=0.6$ and $H=4$ is plotted in Fig. 8.

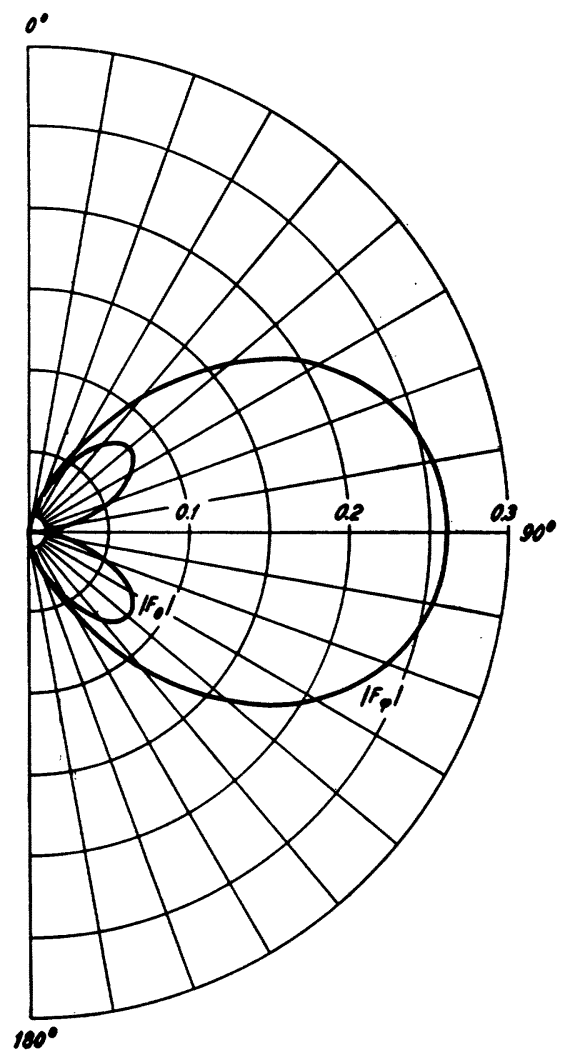

Fig. 8-The $\theta$ - and $\phi$-components of the field radiated by a homogeneous ring quasi-array of radial dipoles with $a / \lambda=0.6$ and $H=4$.

As in the previous section we may express the radiation resistance $R_{H}$ of the quasi-array in relation to the current $I$ in one of the dipoles by (12) and (13), where in the present case

$$
M_{H}(k a)=\frac{1}{2} \int_{0}^{\pi} m_{H}(k a, \theta) \sin \theta d \theta
$$

with $m_{H}(k a, \theta)$ defined in accordance with (10)

$$
\begin{aligned}
m_{H}(k a, \theta) & =F_{\theta} \widetilde{F}_{\theta}+F_{\phi} \widetilde{F}_{\phi} \\
& =\left(J_{H}^{\prime}(z) \cos \theta\right)^{2}+\left(\frac{H}{z} J_{H}(z)\right)^{2} .
\end{aligned}
$$

The gain $g(\theta)$ of the quasi-array considered here is expressed by (16), where $m_{H}(k a, \theta)$ and $M_{H}(k a)$ are given by (26) and (25).

We have to investigate the cases $H=0$ and $|H| \geqq 1$ separately.

$H=0$ : For $H=0$ we find from (26)

$$
m_{0}(k a, \theta)=J_{1}^{2}(z) \cos ^{2} \theta,
$$

and by substituting this expression in (25) we obtain

$$
M_{0}(k a)=\frac{1}{2} \int_{0}^{\pi} J_{1}^{2}(k a \sin \theta) \cos ^{2} \theta \sin \theta d \theta .
$$

As shown in Appendix III this integral may be expressed by

$$
M_{0}(k a)=\frac{1}{8}\left[A_{1}(k a)-B_{1}(k a)\right],
$$

where $A_{n}(x)$ and $B_{n}(x)$ are previously defined functions, expressed in (22) and (23) by tabulated functions for an arbitrary integer index $n$. The function $M_{0}(x)$ is plotted in Fig. 9 for $0 \leqq x \leqq 5$.

$|H| \geqq 1$ : As the radiation resistance and the gain assume the same values for $H$ as for $-H$, it is sufficient to carry out the calculation for $H \geqq 1$. For this range of $H$ we transform the expression (26) for $m_{H}(k a, \theta)$ by using Bessel's recursion formulas; substituting the result in (25) we obtain

$$
\begin{aligned}
M_{H}(k a)= & \frac{1}{8} \int_{0}^{\pi}\left[\left(J_{H-1}(k a)-J_{H+1}(k a)\right)^{2} \cos ^{2} \theta\right. \\
& \left.+\left(J_{H-1}(z)+J_{H+1}(z)\right)^{2}\right] \sin \theta d \theta .
\end{aligned}
$$

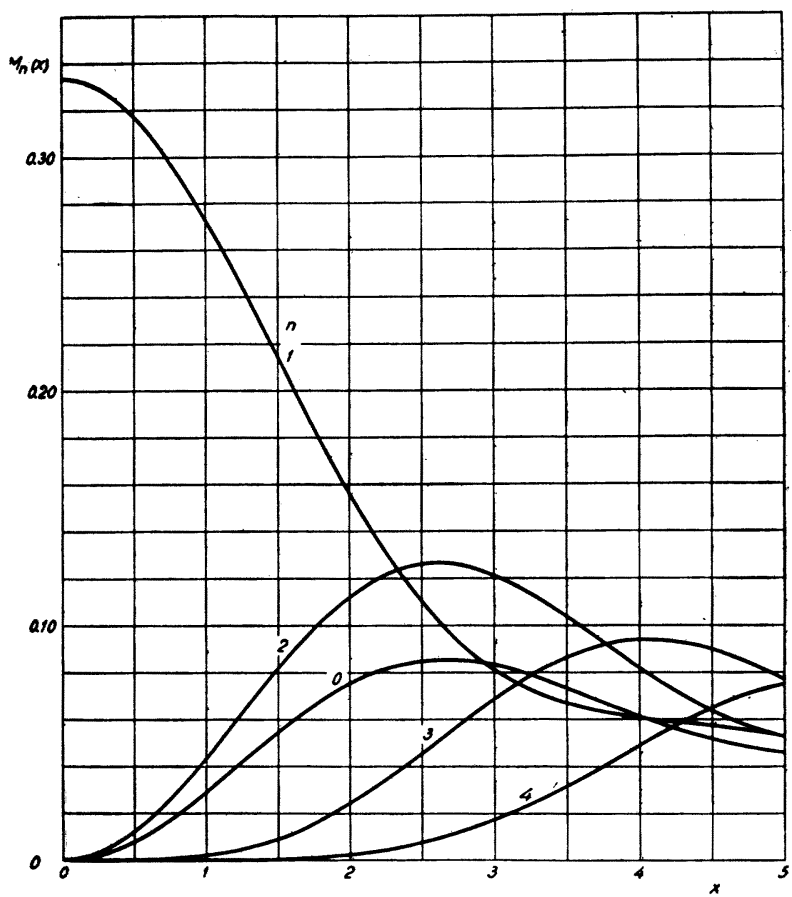

Fig. 9-The function $M_{n}(x)$ defined in (30) for a ring quasi-array of radial dipoles.

Applying the functions $A_{n}(x)$ and $B_{n}(x)$ defined in (22) and (23) we may transform this expression into

$$
M_{H}(k a)=\frac{1}{8}\left[A_{H-1}(k a)+A_{H+1}(k a)+2 B_{H}(k a)\right] .
$$

On the basis of the curves of $A_{n}(x)$ and $B_{n}(x)$ plotted in Appendix II the auxiliary function $M_{n}(x)$ is calculated and plotted in Fig. 9 for $0 \leqq x \leqq 5$ and for $n=0,1,2,3$, and 4. 


\section{Discussion of the Result}

Substituting the above developed expressions for $M_{n}(k a)$ in (12) we find the radiation resistance $R_{H}$ for the ring quasi-array considered here with the current in an arbitrary dipole as reference current. In Fig. 10 $(2 \pi a / s L)^{2} R_{H}$ is plotted as a function of $a / \lambda$ for $|H|=0$, $1,2,3$, and 4 . This figure shows that the radiation resistance increases, though not monotonously, with in-

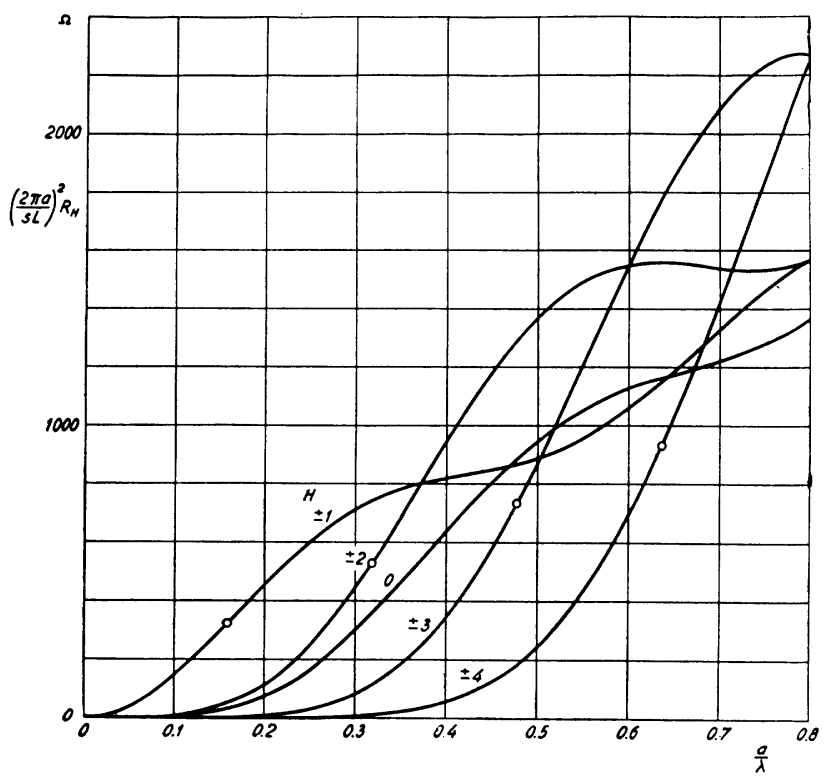

Fig. 10-The radiation resistance $R_{H}$ multiplied by $(2 \pi a / s L)^{2}$ of a homogeneous ring quasi-array of radial dipoles.

creasing radius of the circle. Excepting the case $H=0$ we see that for a small radius of the circle the radiation resistance decreases with increasing $|H|$. Comparing the result arrived at here with the radiation resistance for a corresponding ring quasi-array of tangential dipoles, plotted in Fig. 4, we observe that the radiation resistances for two quasi-arrays with the same radius and with the same $|H|$, but with respectively tangential and radial dipoles, may be very different.

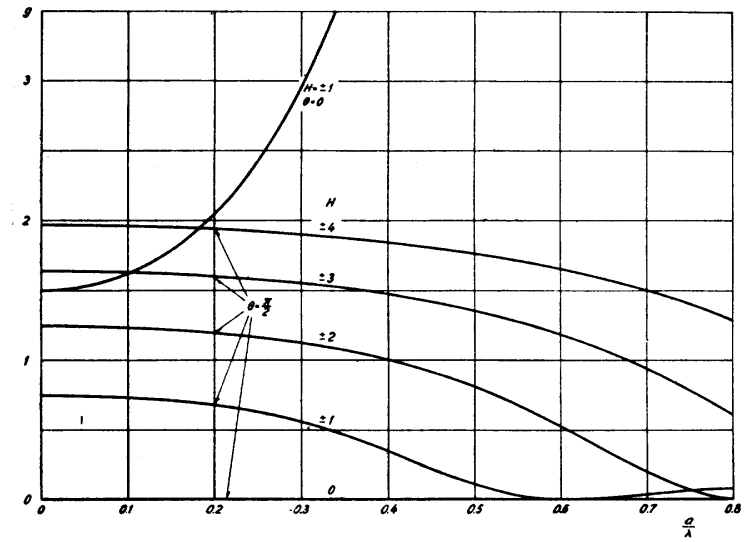

Fig. 11-The gain $g_{H}$ in horizontal directions of a homogeneous ring quasi-array of radial dipoles. The figure also shows the gain in the vertical direction of a quasi-array of this type with $|H|=1$.

The gain of the quasi-array, $g_{H}(\theta)$, is calculated by substituting in (14) the expressions (26), (29) and (31) for $m_{H}(k a, \theta)$ and $M_{H}(k a)$. In Fig. 11 the gain in hori- zontal directions $\theta=\pi / 2$ is plotted as a function of $a / \lambda$ for $|H|=1,2,3$, and 4 . For $H=0$ the gain in horizontal directions is zero. For $|H|=1$ the gain in the directions of the axis, $\theta=0$ and $\pi$, is plotted in Fig. 11 and better in Fig. 12. For other values of $H$ the gain in these directions is zero. The curves of the gain in horizontal directions have the same character as the corresponding curves in the case of tangential dipoles given in Fig. 5; but the present curves decrease more slowly towards the first zero than those in Fig. 5. A homogeneous ring quasi-array of radial dipoles and with $|H| \geqq 2$ seems therefore more fit for obtaining simultaneously a large gain and a large radiation resistance than a corresponding quasi-array of tangential dipoles. In the interval of $a / \lambda$, for which the gain is plotted here, the gain in the direction of the axis assumes much larger values for radial than for tangential dipoles, as is seen by comparing Figs. 6 and 12.

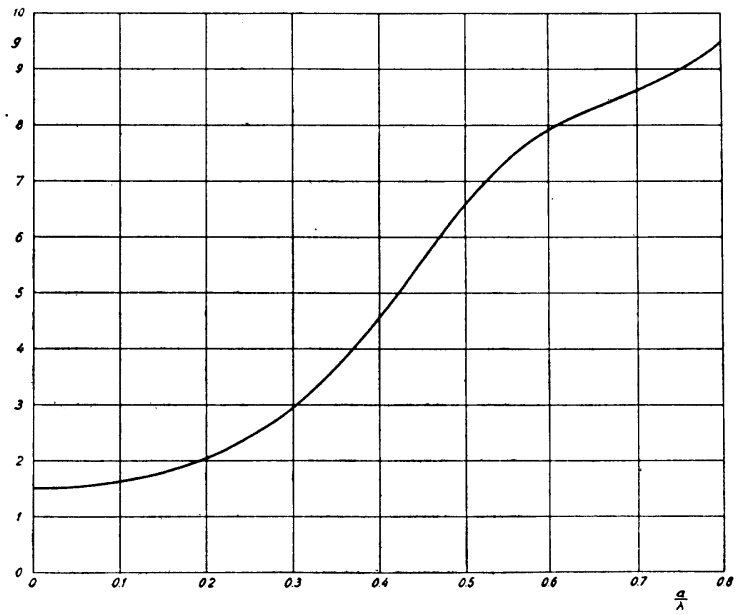

Fig. 12-The gain $g_{1}$ in the vertical direction of a homogeneous ring quasi-array of radial dipoles with $|H|=1$.

\section{Appendix I}

Calculation of $\bar{J}_{n}(x)$

It seems that only few tables of the function

$$
\bar{J}_{n}(x)=\int_{0}^{x} J_{n}(t) d t
$$

have been published. Apart from isolated values it has not been possible for the author to find any table of $\bar{J}_{n}(x)$ for other values of $n$ than $n=0$.

In his monograph on the Bessel functions Watson ${ }^{9}$ gives a table of $\frac{1}{2} \bar{J}_{0}(x), 7 d, x=0(0.02) 1$. A similar table, but calculated to four decimal places only, has been published by Glazenap. ${ }^{10}$

The most comprehensive table of $\bar{J}_{0}(x)$ published so far seems to be a table calculated by Lowan and Abramowitz; this table is characterized by $10 d$, $x=0(0.02) 10$.

${ }^{9}$ G. N. Watson, "A treatise on the theory of Bessel functions," Cambridge England, 2nd ed., p. 752; 1944.

${ }^{10}$ S. P. Glazenap, "Matematicheskie i Astronomicheskie Tablitsy," Leningrad, Russia, pp. 91-115; 1932. 
The reason why no table of $\bar{J}_{n}(x)$ for other values of $n$ than $n=0$ has been published is probably that for $n \geqq 1$ the function $\bar{J}_{n}(x)$ may be expressed through Bessel functions and-in the case of even $n$-the function $\bar{J}_{0}(x)$. As it may be convenient to have a table of tgx, though tables of $\cos x$ and $\sin x$ are available, it will to a still higher degree be convenient to dispose of a table of $\bar{J}_{n}(x)$ for $n \geqq 1$, though $\bar{J}_{n}(x)$ may be expressed by tabulated functions.

To obtain a formula that expresses $\bar{J}_{n}(x)$ through tabulated functions we integrate Bessel's recursion formula expressed as follows

$$
J_{n}(x)=J_{n-2}(x)-2 J^{\prime}{ }_{n-1}(x) .
$$

Hereby we find for $n \geqq 2$

$$
\bar{J}_{n}(x)=\bar{J}_{n-2}(x)-2 J_{n-1}(x) .
$$

As

$$
\bar{J}_{1}(x)=1-J_{0}(x),
$$

we find from this recursion formula

$$
\bar{J}_{n}(x)=\left\{\begin{array}{l}
1-J_{0}(x)-2 \sum_{j=1}^{(n-1) / 2} J_{2 j}(x) \text { for } n \text { odd, } \\
\bar{J}_{0}(x)-2 \sum_{j=1}^{n / 2} J_{2 j-1}(x) \quad \text { for } n \text { even. }
\end{array}\right.
$$

Instead of the formulas given above we may use the following infinite series ${ }^{11}$

$$
\bar{J}_{n}(x)=2 \sum_{j=0}^{\infty} J_{n+2 j+1}(x) .
$$

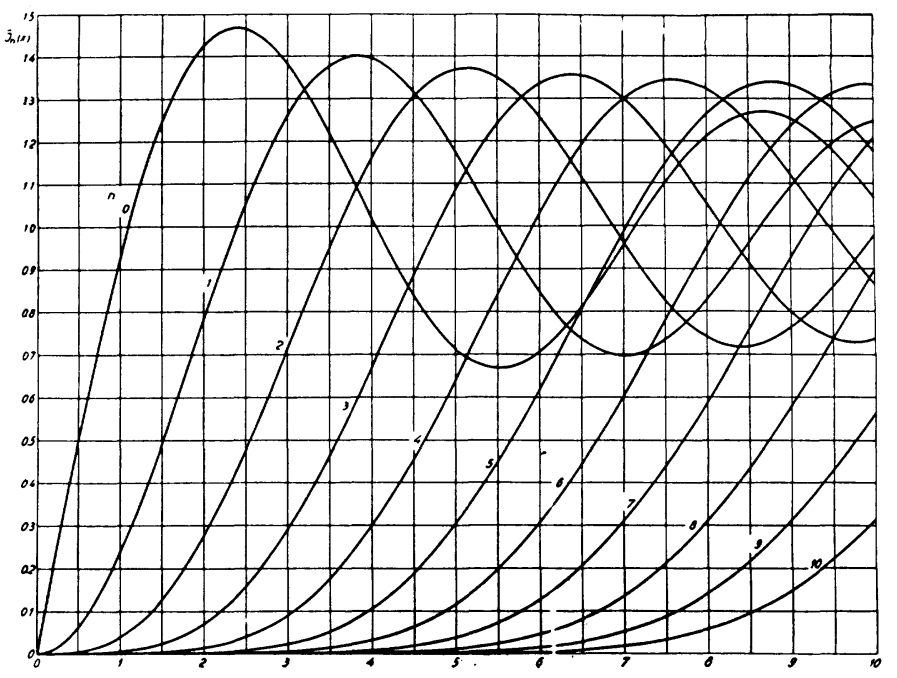

Fig. 13-The function $\bar{J}_{n}(x)=\int_{0}^{x} J_{n}(t) d t$.

As a Bessel function of a constant argument decreases rapidly towards zero with increasing order, this series converges rapidly for $n>x$.

By applying the formulas (36a and b) and by using the tables of the Bessel functions by the Harvard Com-

${ }^{11}$ E. Jahnke and F. Emde, "Funktionentafeln," Leipzig and Berlin, Germany, 3rd ed., p. 145; 1938. putation Laboratory ${ }^{12}$ as well as Lowan and Abramowitz's table of $\bar{J}_{0}(x),{ }^{5}$ a table has been prepared of $\bar{J}_{n}(x)$ for $n=1,2,3, \cdots, 8$ and with $5 d, x=0(0.02) 10 .^{7}$ There is an inaccuracy of one on the last decimal place of the figures in this table. The function $\bar{J}_{n}(x)$ is plotted in Fig. 13 as a function of $x$ for $n=0,1,2, \cdots, 8$ and $0 \leqq x \leqq 10$.

\section{APPENDIX II}

Calculation of the Definite Integrals $A_{n}(x)$ and $B_{n}(x)$

We shall here express the definite integrals $A_{n}(x)$ and $B_{n}(x)$, defined in (20) and (21), by known functions, assuming that $n \geqq 0$.

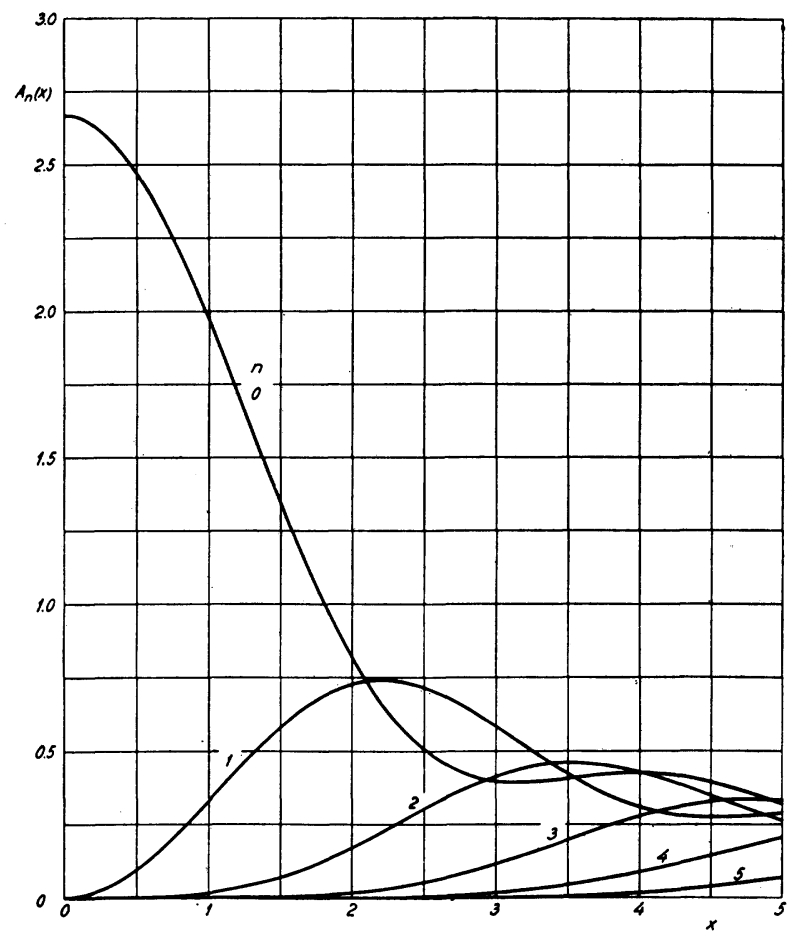

Fig. 14-The function $A_{n}(x)=\int_{0}^{\pi} J_{n}^{2}(x \sin \theta)\left(2-\sin ^{2} \theta\right) \sin \theta d \theta$.

For the purpose of transforming the expression for $A_{n}(x)$ we use Schläfli's series expansion of the product of two Bessel functions ${ }^{13}$

$$
\begin{aligned}
& J_{\mu}(x) J_{\nu}(x) \\
&=\sum_{m=0}^{\infty} \frac{(-1)^{m}\left(\frac{x}{2}\right)^{2 m+\mu+\nu} \Gamma(2 m+\mu+\nu+1)}{m ! \Gamma(m+\mu+\nu+1) \Gamma(m+\mu+1) \Gamma(m+\nu+1)} .
\end{aligned}
$$

Setting $\mu=\nu=n$ in this equation and inserting the resulting expression in (20), we find

$$
\begin{aligned}
A_{n}(x)= & \sum_{n=0}^{\infty} \frac{(-1)^{m}\left(\frac{x}{2}\right)^{2 m+2 n} \Gamma(2 m+2 n+1)}{m ! \Gamma(m+2 n+1) \Gamma^{2}(m+n+1)} \\
& \cdot \int_{0}^{\pi}\left(2-\sin ^{2} \theta\right)(\sin \theta)^{2 m+2 n+1} d \theta .
\end{aligned}
$$

12 "Tables of the Bessel functions of the first kind," Annals of the Computation Laboratory of Harvard University; 1947.

${ }^{13}$ G. N. Watson, op. cit., p. 147. 
We may transform the integrals in the terms of this series expression by using Euler's formula ${ }^{14}$

$$
\int_{0}^{\pi / 2} \cos ^{m-1} x \sin ^{n-1} x d x=\frac{1}{2} \frac{\Gamma\left(\frac{m}{2}\right) \Gamma\left(\frac{n}{2}\right)}{\Gamma\left(\frac{m+n}{2}\right)} .
$$

Setting in this equation $m-1=n-1=p$, we obtain

$$
\begin{aligned}
2^{p+1} \int_{0}^{\pi / 2} \cos ^{p} x \sin ^{p} x d x & =\int_{0}^{\pi / 2} \sin ^{p} 2 x d(2 x) \\
& =\int_{0}^{\pi} \sin ^{p} y d y \\
& =2^{p} \frac{\Gamma^{2}\left(\frac{p+1}{2}\right)}{\Gamma(p+1)} .
\end{aligned}
$$

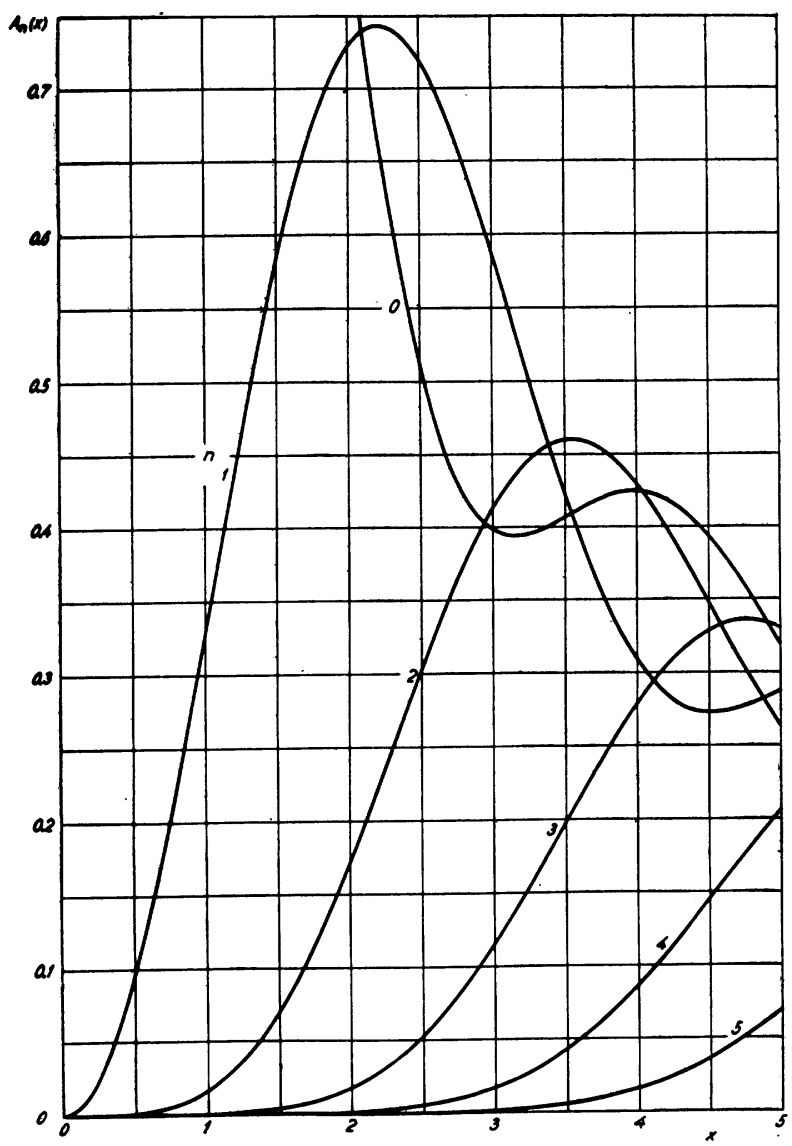

Fig. 15-The function $A_{n}(x)=\int_{0}^{\pi} J_{n}^{2}(x \sin \theta)\left(2-\sin ^{2} \theta\right) \sin \theta d \theta$. This figure shows in greater detail a part of Fig. 14 .

By using this integral we may transform the integral occurring in the series (39) for $A_{n}(x)$

$\int_{0}^{\pi}\left(2-\sin ^{2} \theta\right)(\sin \theta)^{2 m+2 n+1} d \theta$

${ }^{14} \mathrm{E}$. T. Whittaker and G. N. Watson, "A course of modern analysis," Cambridge, England, 4th ed., p. 256; 1940.

$$
\begin{aligned}
= & 2^{2 m+2 n+2} \frac{\Gamma^{2}(m+n+1)}{\Gamma(2 m+2 n+2)} \\
& -2^{2 m+2 n+3} \frac{\Gamma^{2}(m+n+2)}{\Gamma(2 m+2 n+4)} \\
= & 2^{2 m+2 n+2} \frac{\Gamma^{2}(m+n+1)(2 m+2 n+2)(m+n+2)}{\Gamma(2 m+2 n+4)} .
\end{aligned}
$$

Inserting this expression in (39), we find

$$
A_{n}(x)=\sum_{m=0}^{\infty} K_{m} x^{2 m+2 n} \frac{4(m+n+2)}{(2 m+2 n+1)(2 m+2 n+3)},
$$

where, for the sake of brevity, we have introduced

$$
K_{m}=\frac{(-1)^{m}}{m ! \Gamma(m+2 n+1)} .
$$

In transforming the expression for the function $B_{n}(x)$ defined in (21) we proceed in a similar way. In the series expansion (38) for the product of two Bessel functions we set $\mu=n-1$ and $\nu=n+1$. Substituting the resulting expression in (21), we find

$$
\begin{aligned}
B_{n}(x)= & \sum_{m=0}^{\infty} \frac{(-1)^{m}\left(\frac{x}{2}\right)^{2 m+2 n} \Gamma(2 m+2 n+1)}{m ! \Gamma(m+2 n+1) \Gamma(m+n) \Gamma(m+n+2)} \\
& \cdot \int_{0}^{\pi}(\sin \theta)^{2 m+2 n+3} d \theta .
\end{aligned}
$$

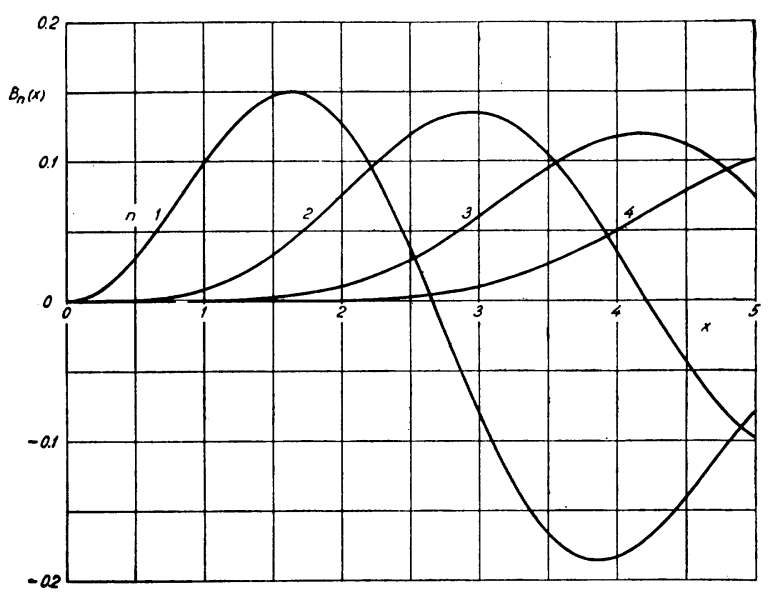

Fig. 16-The function $B_{n}(x)=\int_{0}^{\pi} J_{n-1}(x \sin \theta) J_{n+1}(x \sin \theta) \sin ^{3} \theta d \theta$.

We transform the integrals occurring in this series expansion by using the formula (41) in which we set $p=2 m+2 n+3$,

$\int_{0}^{\pi}(\sin \theta)^{2 m+2 n+3} d \theta=2^{2 m+2 n+3} \frac{\Gamma^{2}(m+n+2)}{\Gamma(2 m+2 n+4)}$.

Inserting this in (45) above for $B_{n}(x)$, we obtain

$$
B_{n}(x)=\sum_{m=0}^{\infty} K_{m} x^{2 m+2 n} \frac{4(m+n)}{(2 m+2 n+1)(2 m+2 n+3)},
$$

where $K_{m}$ is the coefficient defined above in (44). 
The functions $A_{n}(x)$ and $B_{n}(x)$ may be expressed by the Bessel function of the order $2 n$ and the argument $2 x, J_{2 n}(2 x)$, and the integral of this function, $\bar{J}_{2 n}(2 x)$, where we have introduced the notation

$$
\bar{J}_{n}(x)=\int_{0}^{x} J_{n}(t) d t .
$$

The series expansion of the Bessel function $J_{2 n}(2 x)$ is given by

$$
J_{2 n}(2 x)=\sum_{m=0}^{\infty} K_{m} x^{2 m+2 n},
$$

where $K_{m}$ is the coefficient defined in (44). By inspection of the formulas for $A_{n}(x)$ and $B_{n}(x)$ we find that these functions may probably be expressed as a linear combination of the following four functions

$$
\begin{aligned}
& \frac{1}{x} \bar{J}_{2 n}(2 x)=\sum_{m=0}^{\infty} K_{m} x^{2 m+2 n} \frac{2}{2 m+2 n+1}, \\
& \frac{1}{x^{3}} \bar{J}_{2 n}(2 x) \\
& =\sum_{m=-1}^{\infty} K_{m} x^{2 m+2 n} \frac{-2}{(m+1)(m+2 n+1)(2 m+2 n+3)}, \\
& \frac{1}{x^{2}} J_{2 n}(2 x)=\sum_{m=-1}^{\infty} K_{m} x^{2 m+2 n} \frac{-1}{(m+1)(m+2 n+1)}, \\
& \frac{1}{x} J_{2 n+1}(2 x)=\sum_{m=0}^{\infty} K_{m} x^{2 m+2 n} \frac{1}{m+2 n+1} .
\end{aligned}
$$

We set tentatively

$$
\begin{aligned}
& \left.\begin{array}{l}
A_{n}(x) \\
B_{n}(x)
\end{array}\right\}=\left(\frac{a}{x}+\frac{b}{x^{3}}\right) \bar{J}_{2 n}(2 x)+\frac{c}{x^{2}} J_{2 n}(2 x) \\
& +\frac{d}{x} J_{2 n+1}(2 x) \text {. }
\end{aligned}
$$

As the terms corresponding to $m=1$ must disappear, the following relation must be satisfied

$$
2 b=-c[2 m+2 n+3]_{m=-1}=-c(2 n+1) .
$$

Comparing the series expansions of both sides of (54) term by term, we find for the function $A_{n}(x)$

$$
a=\frac{3}{2}, \quad b=-\frac{4 n^{2}-1}{8}, \quad d=-\frac{1}{2},
$$

from which we obtain

$$
\begin{gathered}
A_{n}(x)=\left(\frac{3}{2 x}-\frac{4 n^{2}-1}{8 x^{3}}\right) \bar{J}_{2 n}(2 x)+\frac{2 n-1}{4 x^{2}} J_{2 n}(2 x) \\
-\frac{1}{2 x} J_{2 n+1}(2 x) .
\end{gathered}
$$

For the function $B_{n}(x)$ we find by comparing both sides of (54) term by term

$$
a=-\frac{1}{2}, \quad b=\frac{3}{8}\left(4 n^{2}-1\right), \quad d=\frac{3}{2},
$$

from which we obtain

$$
\begin{aligned}
B_{n}(x)= & \left(-\frac{1}{2 x}+\frac{3\left(4 n^{2}-1\right)}{8 x^{3}}\right) \bar{J}_{2 n}(2 x) \\
& -\frac{3(2 n-1)}{4 x^{2}} J_{2 n}(2 x)+\frac{3}{2 x} J_{2 n+1}(2 x) .
\end{aligned}
$$

\section{APPENDIX III}

\section{Calculation of the Definite Integral $M_{0}(x)$ Defined for} Radial Dipoles

In this section we shall express the function $M_{0}(x)$ defined for radial dipoles in (28). For the square of the Bessel function in this formula we substitute the series expansion (38) for the product of two Bessel functions in which we set $\nu=\mu=1$. Hereby we obtain

$$
\begin{aligned}
M_{0}(x)= & \frac{1}{2} \sum_{m=0}^{\infty} \frac{(-1)^{m}\left(\frac{x}{2}\right)^{2 m+2} \Gamma(2 m+3)}{m ! \Gamma(m+3) \Gamma^{2}(m+2)} \\
& \cdot \int_{0}^{\pi}\left[(\sin \theta)^{2 m+3}-(\sin \theta)^{2 m+5}\right] d \theta .
\end{aligned}
$$

By using (41) we find

$$
\begin{aligned}
\int_{0}^{\pi}\left[(\sin \theta)^{2 m+3}-(\sin \theta)^{2 m+5}\right] d \theta & \\
= & 2^{2 m+3} \frac{\Gamma^{2}(m+2)}{\Gamma(2 m+4)}-2^{2 m+5} \frac{\Gamma^{2}(m+3)}{\Gamma(2 m+6)} \\
= & 2^{2 m+4} \frac{\Gamma^{2}(m+2)(m+2)}{\Gamma(2 m+6)} .
\end{aligned}
$$

Inserting this result in (60) we obtain

$$
M_{0}(x)=\sum_{m=0}^{\infty} \frac{(-1)^{m} x^{2 m+2}}{m ! \Gamma(m+3)(2 m+3)(2 m+5)} .
$$

By comparing this expression for $M_{0}(x)$ with the series expressions (43) and (47) for $A_{n}(x)$ and $B_{n}(x)$ we see that

$$
M_{0}(x)=\frac{1}{8}\left[A_{1}(x)-B_{1}(x)\right]
$$

\section{ACKNOWLEDGEMENT}

The author wishes to express his thanks to the head of The Microwave Laboratory, Professor J. Oskar Nielsen, for his continual encouragement, and to Professor J $\phi$ rgen Rybner for having suggested a simplified derivation of the formulas in Appendixes II and III. I am also indebted to Mr. Preben Gudmandsen for valuable criticism. The English translation was revised by Mr. Oscar Kasch, sworn translator and interpreter. 